\title{
Pulverizador Agrícola em Barras com Aplicação a Taxa Variada: Modelagem Física
}

\author{
Gabrielle R. A. Rosa* Fábio P. Terra ${ }^{*, * *}$ \\ Paulo L. J. Drews Jr* \\ * NAUTEC - Grupo de Automação e Robótica Inteligentes, C3, \\ Universidade Federal do Rio Grande, RS, (e-mail: \\ gabriellerar@gmail.com, paulodrews@furg.br). \\ ** IFSul - Instituto Federal Sul-Rio-Grandense, Pelotas, RS, (e-mail: \\ fabioterra@gmail.com)
}

\begin{abstract}
Agriculture has undergone changes in order to reduce the labor force and increase the productivity due to the technological advance. In this context, pesticide application has been revolutionized with the use of intelligent sprayers able to control the places where the spraying is really necessary. Some companies in the market sell solutions to meet these requirements, but the cost is sometimes so high as to make their use prohibitive by small production properties. Therefore, this paper aims to perform the mathematical modeling to allow pressure and flow control along a boom sprayer with nozzle application and serial feed, keeping process variables stable. Thereafter, this work is part of a project that aims to develop a low-cost solution of precision agriculture available for Smallholders and Family Farming.
\end{abstract}

Resumo: A agricultura tem sofrido inúmeras mudanças devido ao avanço tecnológico, permitindo aumento de produtividade e redução de mão de obra no campo. Nesse contexto, revolucionou-se a aplicação de agrodefensivos com o uso de pulverizadores capazes de controlar os locais onde de fato é necessária a pulverização. Inclusive, existem empresas abordando soluções comerciais com estas características. Todavia, o alto custo destes equipamentos viabiliza seu uso apenas por agricultores de grande porte. O trabalho visa, então, realizar a modelagem matemática de um sistema de pulverização, avaliando a relação entre pressão e vazão ao longo de uma barra, no intuito de permitir o controle da aplicação bico a bico, mantendo as variáveis de processo constantes. Este é parte de um projeto de desenvolvimento de soluções de agricultura de precisão, de baixo custo, para otimizar a aplicação de defensivos agrícolas via pulverizadores, atendendo às necessidades das pequenas propriedades rurais, no contexto da agricultura familiar.

Keywords: Modeling; Pressure Control; Fluidic System; Boom Sprayer; Precision Agriculture. Palavras-chave: Modelagem; Controle de Pressão; Sistema Fluídico; Pulverizador em Barras; Agricultura de Precisão.

\section{INTRODUÇÃO}

A introdução de novas tecnologias para a produção de commodities agrícolas a partir da década de 1950, acarretou em um aumento expressivo da carga de agroquímicos utilizada no controle de pragas nas plantações. Devido a essas profundas mudanças que a agricultura tem sofrido, de acordo com o Instituto Nacional do Câncer (INCA), o consumo médio de agroquímicos na produção agrícola do país equivale a 5 litros de agrotóxico por ano por habitante (Milhorance, 2015).

Outro fator a ser considerado é que o problema também pode causar poluição ambiental e desequilíbrio do agrossistema (Grützmacher et al., 2008). Prova disso é que, em alguns casos, em função da deriva, até $99,9 \%$ do produto aplicado pode se mover para locais como águas superficiais e subterrâneas (Ribeiro et al., 2007). Ainda, de acordo com dados de 2017 divulgados pelo IBGE, no Estado do Rio Grande do Sul, aproximadamente $70 \%$ dos produtores utilizam agrodefensivos. Tomando como exemplo a cultura da cebola, embora o Estado possua aproximadamente 31 mil estabelecimentos (IBGE, 2017), sua produção é baixa, quando comparada à de outros Estados do território nacional. Conclui-se, portanto, que a maioria dos produtores é de pequeno porte e poderia ser beneficiado com a aplicação da solução desenvolvida a partir da modelagem proposta.

Tendo em vista o problema supracitado, no que tange ao uso de pulverizadores em barras (Figura 1), a agricultura de precisão apresenta algumas possíveis soluções como o controle por seções das linhas de pulverização, conforme pode ser visto na Figura 2.

Esta tecnologia é discutida em Felizardo et al. (2016) e utilizada por algumas empresas, como é o caso da John Deere, que defende que a automatização do processo de abertura e fechamento das seções da barra reduz a fadiga do operador e a sobreposição do produto aplicado. Consequentemente, acaba por reduzir os custos e os impactos ambientais agregados (Deere, 2018). 


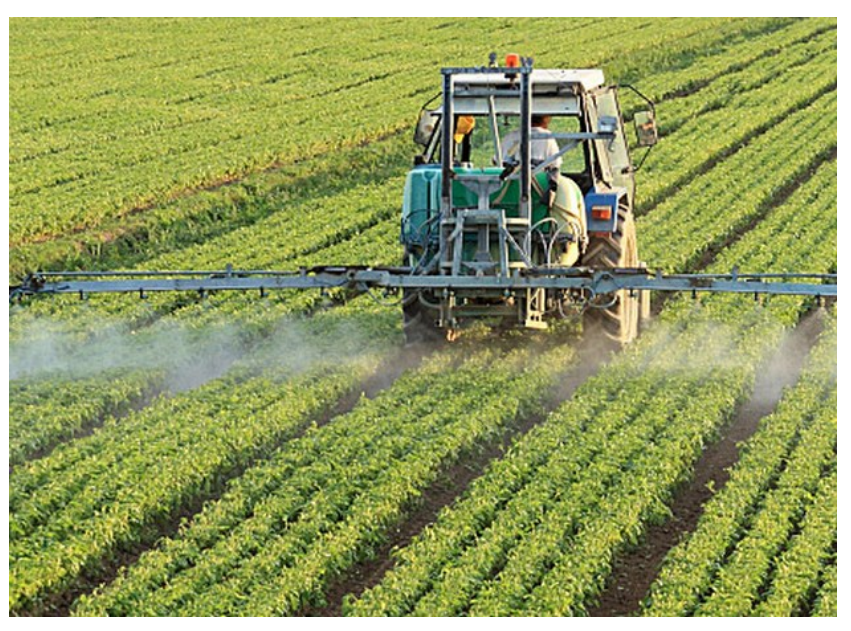

Figura 1. Pulverizador em barras tratorizado Newlands (2019)

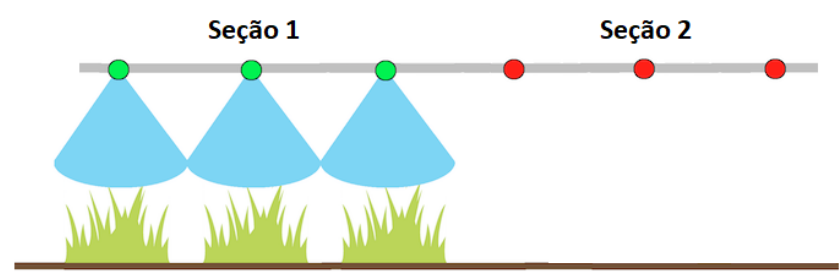

Figura 2. Controle por seções de pulverizador em barras. As cores verde e vermelho representam os bicos abertos ou fechados, respectivamente

Sobremaneira, ainda que o sistema de seleção por seções apresente vantagens em relação aos sistemas convencionais, isto é, sistemas sem qualquer tipo de controle ou automação, o mesmo não supre a carência no que se refere a linhas de plantação com trechos de tamanhos inferiores aos das seções do pulverizador e também nos casos onde há falhas de continuidade nas próprias linhas das lavouras.

Sendo assim, surge o conceito de controle bico a bico, apresentado na Figura 3, no qual cada ponta de pulverização é controlada individualmente de acordo com a necessidade específica da plantação.

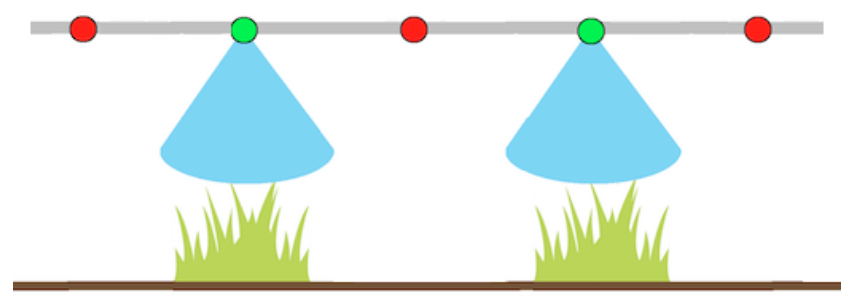

Figura 3. Controle bico a bico de pulverizador em barras. Cores verde e vermelho representam bicos abertos ou fechados, respectivamente

O controle bico a bico abordado na Figura 3 pode ser, ainda, dividido em dois tipos, série ou paralelo, de acordo com a alimentação de produto no mesmo. Dessa forma, nos sistemas paralelos cada bico de pulverização possui conexão própria com o tanque de armazenamento do fluido, diferentemente dos sistemas em série, em que uma mesma tubulação é responsável por alimentar diversos bicos da barra do pulverizador, em uma configuração típica de linha tronco (header).

Nesse contexto, Mercaldi et al. (2017) assume que a distribuição de pressão é constante e que, portanto, não há perda de carga ao longo das mangueiras da barra de pulverização. Sabe-se, porém, que existe perda de pressão em virtude da própria tubulação e também devido à passagem pelas válvulas de acionamento dos bicos (Delmée, 2003).

É importante destacar que as variáveis de pressão e vazão são importantes uma vez que ambas estão relacionadas mutuamente e um aumento de pressão causa problema no formato da gota do produto aplicado. Em outras palavras, pode-se dizer que cada gota, ao passar pelo bico pulverizador, gera uma população de gotas com diâmetro médio tal que possa combater o respectivo alvo biológico. Por outro lado, o descontrole do tamanho da gota afeta diretamente a qualidade da aplicação do defensivo agrícola (Holterman et al., 2016).

Novamente, há uma solução comercial com esta abordagem, como é o caso do Omni 700, lançado pela empresa Jacto em 2016, e capaz de realizar o controle bico a bico em série (Otmis, 2017). Em contrapartida, o custo de pulverizadores deste porte, com toda a tecnologia embarcada, extrapola a monta de 800 mil reais (Araújo, 2016) e, portanto, representa uma solução economicamente inviável para a agricultura familiar.

Neste contexto, o presente trabalho tem como contribuição a modelagem matemática de uma barra de pulverização agrícola, visando avaliar o comportamento da pressão e da vazão. Com isso, visa-se definir uma estratégia para que a pressão na barra permaneça constante, independente do cenário de abertura ou fechamento dos bicos que a compõem.

\section{MODELO MATEMÁTICO}

O módulo de pulverização é composto por restrições hidráulicas que provocam variação na pressão e, consequentemente, na vazão do sistema. Os principais elementos que se opõem à passagem do fluido são decorrentes do atrito viscoso com as paredes internas da tubulação e das conexões, além das pontas de pulverização e da válvula de controle. Todos eles, portanto, comportam-se como resistências fluídicas (Garcia, 2005).

Conforme Hughes (2002), o perfil de escoamento de um fluido interfere significativamente na relação entre as variáveis de pressão e vazão. Portanto, para avaliar corretamente o comportamento interdependente destas variáveis, é fundamentel a determinação prévia do perfil de escoamento do fluido. Para isso, utiliza-se o número de Reynolds, conforme apresenta a equação (1).

$$
R e=\frac{\rho \cdot d_{i} \cdot v}{\mu},
$$

onde $\rho$ é a densidade absoluta (massa específica) do fluido $\left[\mathrm{kg} / \mathrm{m}^{3}\right] ; d_{i}$ é o diâmetro interno da tubulação pela qual escoa o fluido $[m]$; $v$ é a velocidade média de escoamento 
do fluido $[\mathrm{m} / \mathrm{s}]$; e $\mu$ é a viscosidade dinâmica do fluido $[P a \cdot s]$.

Logo, para Garcia (2005), quando o número de Reynolds for menor que 1100, trata-se de um perfil de escoamento laminar, enquanto valores maiores que 3500 representam escoamento turbulento. Já no caso de valores presentes no intervalo entre esses limites, diz-se que o escoamento tem regime transitório. É importante ressaltar que estas faixas são aproximadas e variam de autor para autor.

Uma vez identificado o regime de escoamento do fluido, se laminar ou turbulento, pode-se modelar corretamente o sistema. Para fins de estimativa do comportamento do sistema, assumiu-se escoamento predominantemente estacionário e foi realizada a modelagem a partir da equação de continuidade de Bernoulli, que pode ser vista na equação (2).

$$
P_{1}+\rho \cdot g \cdot h_{1}+\frac{1}{2} \cdot \rho \cdot v_{1}^{2}=P_{2}+\rho \cdot g \cdot h_{2}+\frac{1}{2} \cdot \rho \cdot v_{2}^{2},
$$

onde $P_{1}, h_{1}$ e $v_{1}$ são a pressão $[P a]$, a altura $[m]$ e a velocidade $[\mathrm{m} / \mathrm{s}]$ à montante, respectivamente; e $P_{2}, h_{2}$ e $v_{2}$ são as variáveis à jusante do elemento hidráulico. Ainda, $\rho$ é a densidade absoluta do fluido $\left[\mathrm{kg} / \mathrm{m}^{3}\right]$ e $g$ é a gravidade local do sistema $\left[\mathrm{m} / \mathrm{s}^{2}\right]$.

Para os elementos hidráulicos do módulo de pulverização, é possível considerar que a diferença de altura entre os pontos 1 e 2 é nula, visto que estes pontos estão alinhados horizontalmente tanto no caso dos trechos de tubulações e válvula quanto no caso dos bicos de pulverização.

Sendo assim, para $h_{1}=h_{2}$, o Teorema de Bernoulli pode ser reduzido, conforme mostrado na equação (3).

$$
P_{1}+\frac{1}{2} \cdot \rho \cdot v_{1}^{2}=P_{2}+\frac{1}{2} \cdot \rho \cdot v_{2}^{2}
$$

Através de simplificações, considerando $P_{2}-P_{1}=\Delta P$ e resolvendo a equação (3) em termos de $v_{1}$, obtém-se a equação (4).

$$
v_{1}^{2}=v_{2}^{2}-\frac{2 \cdot \Delta P}{\rho} .
$$

Por outro lado, com base na Equação da Continuidade, pode-se afirmar que o volume ao longo do escoamento permanece constante, uma vez que se trata de um líquido incompressível e que não há adição nem perda de fluido. Logo, a vazão que entra no trecho de tubo é exatamente a vazão que sai, estabelecendo-se, então, a equação (5).

$$
A_{1} \cdot v_{1}=A_{2} \cdot v_{2}
$$

onde $A_{1}$ é a área $\left[m^{2}\right]$ e $v_{1}$ é a velocidade de escoamento no ponto $1 ;[\mathrm{m} / \mathrm{s}]$ e $A_{2}$ e $v_{2}$ são, respectivamente, a área e a velocidade de escoamento do fluido no ponto 2 .

Logo, resolvendo-se a equação (5) em termos de $v_{2}$ e substituindo-se na equação (4), obtém-se a expressão apresentada na equação (6).

$$
v_{1}=\sqrt{\frac{2 \cdot \Delta P}{\rho \cdot\left[\left(\frac{A_{1}}{A_{2}}\right)^{2}-1\right]}} .
$$

Sabendo que a vazão volumétrica é $Q=A_{1} \cdot v_{1} \mathrm{e}$ substituindo-se $v_{1}$ pela expressão obtida na equação (6), pode-se afirmar que a vazão num trecho de tubo será dada pela equação (7).

$$
Q=A_{1} \cdot \sqrt{\frac{2 \cdot \triangle P}{\rho \cdot\left[\left(\frac{A_{1}}{A_{2}}\right)^{2}-1\right]}},
$$

onde $Q$ é a vazão volumétrica do sistema $\left[\mathrm{m}^{3} / \mathrm{s}\right] ; A_{1}$ e $A_{2}$ são as áreas transversais internas $\left[\mathrm{m}^{2}\right]$ nos pontos 1 e 2 por onde escoa o fluido; $\rho$ é a densidade absoluta do fluido $\left[\mathrm{kg} / \mathrm{m}^{3}\right]$; e $\Delta P$ é a diferença de pressão entres os pontos 1 e $2[\mathrm{~Pa}]$.

De acordo com Garcia (2005), a equação (7) diz respeito à natureza puramente teórica dos elementos hidráulicos, o que não representa de fato um sistema real. Sendo assim, para um sistema real é necessária a inserção do coeficiente de descarga $\left(C_{D}\right)$ do elemento hidráulico.

Tal coeficiente é obtido experimentalmente e é relativo à correção da energia perdida devido à turbulência e ao efeito de "vena contracta", podendo ser calculado conforme a equação (8).

$$
C_{D}=\frac{Q_{r}}{Q_{t}}
$$

Na equação (8), $Q_{r}$ representa a vazão real do elemento hidráulico $\left[\mathrm{m}^{3} / \mathrm{s}\right]$; e $Q_{t}$ sua vazão teórica $\left[\mathrm{m}^{3} / \mathrm{s}\right]$. Logo, para a obtenção da vazão real do escoamento do fluido, deve-se inserir o coeficiente $C_{D}$ na equação $(7)$, resultando na equação (9).

$$
Q=C_{D} \cdot A_{1} \cdot \sqrt{\frac{2 \cdot \Delta P}{\rho \cdot\left[\left(\frac{A_{1}}{A_{2}}\right)^{2}-1\right]}} .
$$

Dessa forma, a equação (9) pode ser resumida em termos da relação entre a vazão e a pressão dos elementos do sistema hidráulico, conforme apresenta a equação (10), onde $R_{F}$ representa a resistência fluídica do elemento hidráulico $\left[k P a /(L / m i n)^{2}\right]$.

$$
\Delta P=R_{F} \cdot Q^{2}
$$

É válido destacar que, uma vez que $1 \cdot \mathrm{m}^{3} / \mathrm{s}$ equivale a $60000 \cdot L / \min$, na equação (10) utilizou-se fator de conversão para alteração da unidade de vazão.

Sendo assim, Felizardo et al. (2016) afirma que a perda de carga em elementos deprimogênios $\left(R_{F, D}\right)$, i. e., pontas de pulverização e válvula, pode ser dada de acordo com a equação (11).

$$
R_{F, D}=\frac{\rho}{2 \cdot C_{D}^{2} \cdot A_{i}^{2}} .
$$

Em que $R_{F, D}$ é dado por $\left[\mathrm{kg} / \mathrm{m}^{7}\right]$; $\rho$ é a densidade absoluta do fluido $\left[\mathrm{kg} / \mathrm{m}^{3}\right], C_{D}$ é o coeficiente de descarga do 
elemento hidráulico; e $A_{i}$ é a área de seção transversal interna de escoamento do fluido $\left[\mathrm{m}^{2}\right]$.

Analogamente, baseado em Garcia (2005), a perda de carga em trechos de tubulações $\left(R_{F, T}\right)$ pode ser calculada através da equação (12), uma vez que o coeficiente de descarga para estes trechos é dado por $C_{D}^{2}=\frac{d_{i}}{f_{a} \cdot L}$.

$$
R_{F, T}=\frac{f_{a} \cdot L \cdot \rho}{2 \cdot d_{i} \cdot A_{i}^{2}}
$$

Na equação (12) $f_{a}$ é o coeficiente de descarga distribuído; $L$ é o comprimento do trecho de tubo $[m] ; \rho$ é a densidade absoluta do fluido $\left[\mathrm{kg} / \mathrm{m}^{3}\right] ; d_{i}$ é o diâmetro interno $[\mathrm{m}]$; e $A i$ é a área interna $\left[\mathrm{m}^{2}\right]$ da tubulação.

Nota-se, nesse momento, a importância da determinação do número de Reynolds devido à sua grande influência no coeficiente de carga distribuído $f_{a}$, obtido a partir do Diagrama de Moody (Bega et al., 2006).

Dessa forma, a barra de pulverização pode ser modelada a partir do conceito de resistência fluídica apresentado por Steward and Humburg (2000) e, portanto, a pressão do sistema é dada pela equação (13).

$$
P_{S}=\left(R_{F, D}+R_{F, T}\right) \cdot Q^{2} .
$$

Com o comportamento do sistema definido a partir do conceito apresentado por Steward and Humburg (2000), a Figura (4) apresenta o Diagrama de Blocos para o modelo obtido.

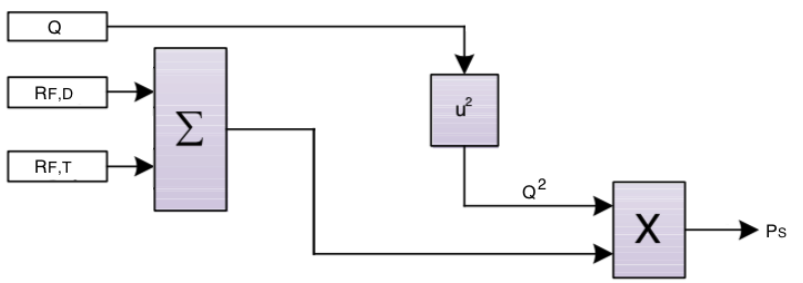

Figura 4. Diagrama de blocos do comportamento do sistema.

Percebe-se, dessa forma, que as variáveis vazão e pressão estão relacionadas e o comportamento de uma reflete diretamente no comportamento da outra. Nesse contexto, é possível destacar a necessidade da inserção da válvula de controle para regulação do fluxo ao longo da linha de pulverização, permitindo maior estabilidade da pressão mesmo nos cenários de variação de consumo de vazão, devido à abertura ou fechamento dos bicos pulverizadores.

\section{RESULTADOS EXPERIMENTAIS}

Os experimentos que serão apresentados a seguir foram realizados no Centro de Ciências Computacionais (C3) da Universidade Federal do Rio Grande (FURG). Para tal, utilizou-se um sistema de pulverização agrícola, adaptado para uso em laboratório, composto por reservatório de 100 litros, bomba de pistão para pulverização, barra de pulverização de cinco (5) metros com sete (7) bicos, conforme pode ser visto nas Figuras 5 e 6 .

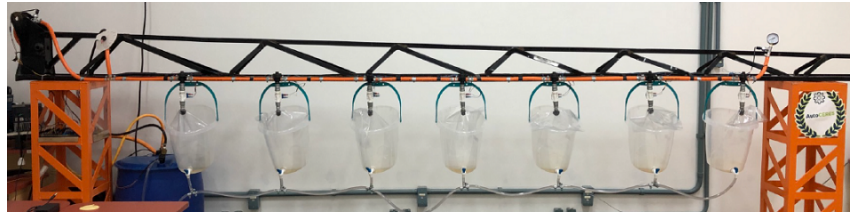

Figura 5. Protótipo - Visão geral da barra de pulverização utilizada para a realização dos experimentos.

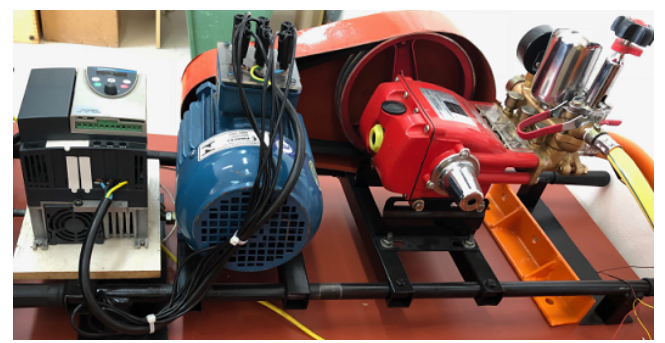

Figura 6. Protótipo - Conjunto motor, bomba e inversor de frequência, utilizado para simular um sistema de tração com trator e de bombeamento.

Como mostrado na Figura 5, no sistema utilizado, foram adicionados medidores eletrônicos de pressão (PT) e vazão (FT), manômetro (PG), válvula reguladora de pressão (PV), além de válvulas solenoides (on/off) para abertura/fechamento de cada um dos bicos de pulverização. Os sensores de vazão adotados são do modelo YF-S201 com medição volumétrica, enquanto os sensores de pressão piezo-resistivos (strain gauge) são do modelo HK1100C.

Na Figura 7, apresenta-se o fluxograma de processo resumido do sistema, com a instrumentação utilizada. Cumpre destacar que a identificação ("tagueamento") dos instrumentos foi realizada com base nos critérios da norma ISA 5.1 (ISA, 2009).

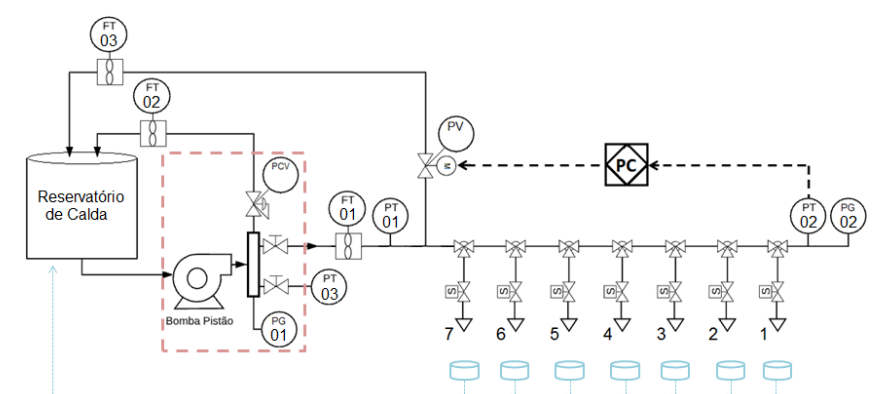

Figura 7. Fluxograma de processo da barra de pulverização.

Nas Figuras 8 e 9 são apresentados os detalhes de instalação tanto da válvula reguladora de pressão (PV), quanto das válvulas solenoides.

A calda (solução aquosa) é propelida do reservatório para os bicos através de uma bomba de deslocamento positivo com vazão nominal de $14 \mathrm{~L} / \mathrm{min}$, alimentando os sete bicos de pulverização instalados em série sobre a barra do pulverizador. Além disso, com medidores de pressão e vazão instalados foram obtidos dados para o monitoramento da dinâmica operacional do sistema.

Os dados dos medidores foram coletados e processados via microcontrolador ATMEL ATmega2560. A partir do 
Tabela 1. Dados obtidos experimentalmente com o fechamento e a abertura dos bicos de pulverização.

\begin{tabular}{cccc}
\hline Bicos abertos & $\Delta P\left[\mathrm{kgf} / \mathrm{cm}^{2}\right]$ & $Q[\mathrm{~L} / \mathrm{min}]$ & $R_{\text {Fequivalente }}$ \\
\hline 7 & 3,57 & 5,60 & 0,114 \\
6 & 3,88 & 5,07 & 0,151 \\
5 & 4,24 & 4,40 & 0,219 \\
4 & 4,64 & 3,60 & 0,358 \\
3 & 4,96 & 2,80 & 0,633 \\
2 & 5,27 & 2,00 & 1,318 \\
1 & 5,58 & 1,00 & 5,580 \\
\hline
\end{tabular}

microcontrolador, foi também possível enviar comandos de abertura ou fechamento para as válvulas solenoides instaladas junto aos bicos de pulverização viabilizando, portanto, que sejam controlados remotamente e de forma rápida. Com isso, foi possível a realização de análises do comportamento do sistema, dadas variadas sequências de fechamento e abertura dos bicos de pulverização. Os bicos utilizados foram do tipo leque 0,2 com $110^{\circ}$ de abertura, da empresa Fan Tip (Tip, 2018).

Logo, a partir da Tabela 1, é possível observar os resultados obtidos para a resistência fluídica equivalente do módulo de pulverização para os bicos em destaque, mantendo-se a válvula de recirculação (HV) fechada. Além disso, para uma pressão de 4,0 bar, o fabricante afirma que a vazão do bico para o uso comercial é de aproximadamente 0,92 $L / \min$, informação essa que se confirma dada a aproximação das condições as quais os bicos foram submetidos.

Além disso, é importante destacar que as medidas de pressão são manométricas, isto é, relativas a pressão atmosférica local e, ainda, que nos ensaios as pressões medidas por PT-01 e PT-02 foram muito próximas, constatando-se que a pressão se distribui uniformemente ao longo da barra de pulverização sob essas condições.

Sendo assim, a relação entre a resistência fluídica equivalente $\left(R_{F \text { equivalente }}\right)$ e a quantidade de bicos abertos no sistema $(n)$ pode ser descrita a partir da equação (14) e, através da Figura 10 é possível observar sua proximidade com os valores obtidos experimentalmente.

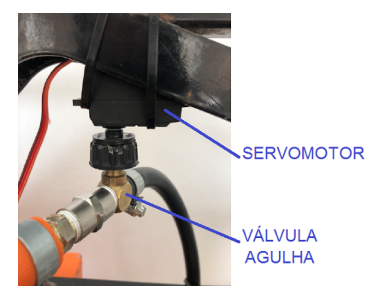

Figura 8. Detalhes de instalação das válvula reguladora de pressão - PV.

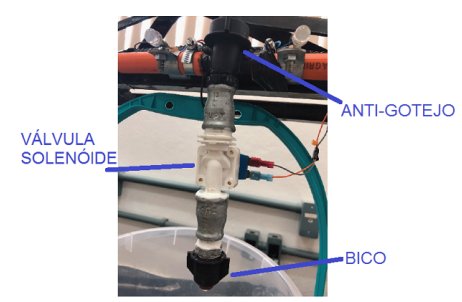

Figura 9. Detalhes de instalação das válvulas do tipo solenoide.

$$
R_{F}(n)=42,59 \cdot e^{-2,335 \cdot n}+2,307 \cdot e^{-0,4591 \cdot n}
$$

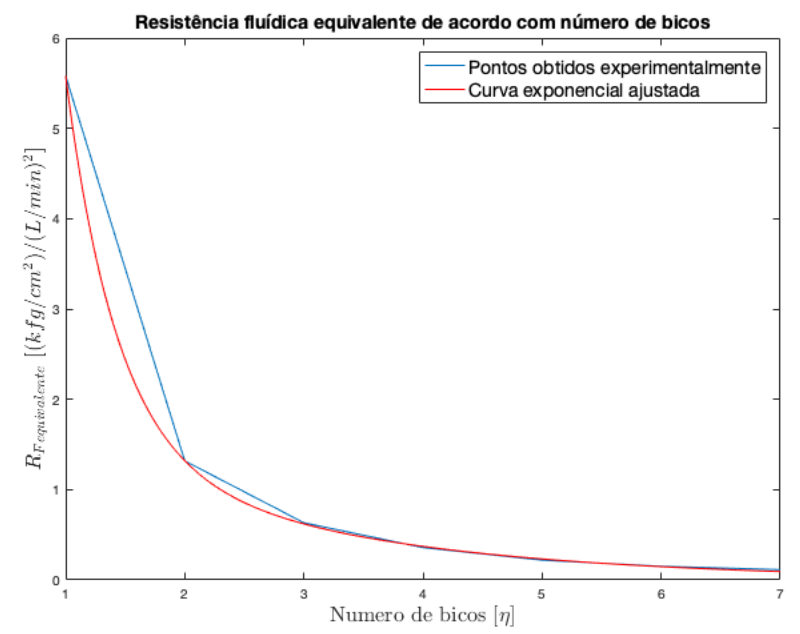

Figura 10. Comparação entre os dados do experimento e a curva teórica obtida. A diferença entre a curva em vermelho e as retas em azul se deve a aproximação retilínea com os pontos feita para visualização.

Um importante fator a ser considerado é o valor do número de Reynolds, cujo comportamento ao longo dos ensaios pode ser visto na Figura 11.

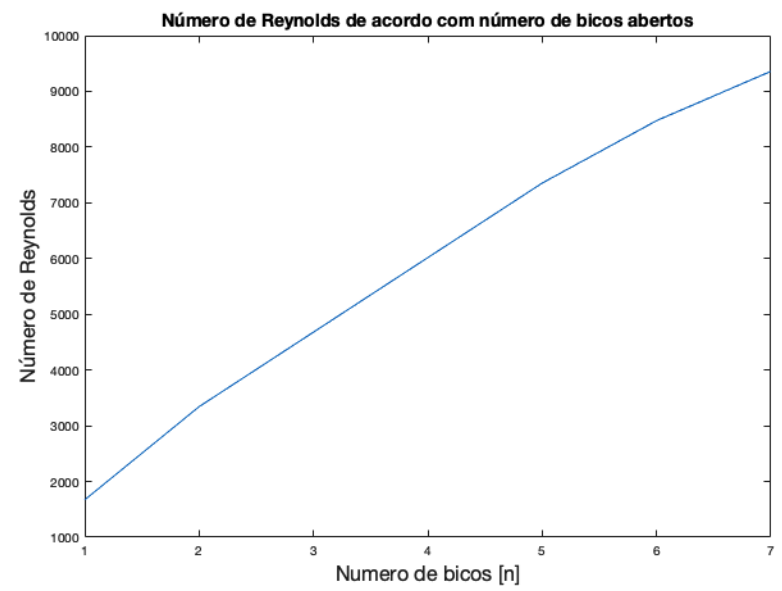

Figura 11. Curva que relaciona a quantidade de bicos abertos com o número de Reynolds.

A partir da Figura 11 destaca-se que o escoamento do fluido passa do perfil laminar para o turbulento. Isto ocorre devido às características construtivas do bico de pulverização, visto que a vazão de consumo individual por bico é extremamente baixa, em comparação com a capacidade de fornecimento da bomba, provocando pequena variação de velocidade do fluido na mangueira (header) para cada cenário.

Na Figura 12, pode-se observar o comportamento da pressão a partir da inclusão da dinâmica de uma válvula reguladora do tipo agulha, com diâmetro nominal de $1 / 4$ ".

No ensaio realizado, apresentado na Figura 12, observase que, com a pressão inicial de $4,00 \mathrm{~kg} / \mathrm{cm}^{2}$ e a válvula PV totalmente fechada, foram fechados simultaneamente os bicos 1, 4 e 7, causando aumento na pressão para um 


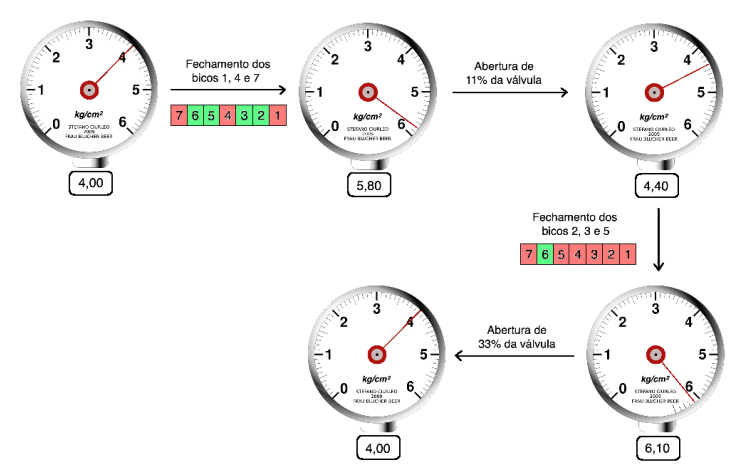

Figura 12. Dinâmica da pressão, modificada pela válvula reguladora.

patamar de $5,80 \mathrm{~kg} / \mathrm{cm}^{2}$. A partir daí, abriu-se $11 \%$ da válvula, trazendo a pressão para $4,40 \mathrm{~kg} / \mathrm{cm}^{2}$. Promovendo o fechamento de mais três bicos (2, 3 e 5$)$, a pressão sobe para $6,10 \mathrm{~kg} / \mathrm{cm}^{2}$. Aumentando a abertura da válvula para $33 \%$, a pressão volta para aproximadamente 4, $00 \mathrm{~kg} / \mathrm{cm}^{2}$. Conclui-se, portanto, que é possível regular a pressão ao longo da barra, a partir da modulação da abertura de uma válvula (PV) instalada de forma a aliviar o excedente de vazão gerado pela bomba.

\section{CONCLUSÕES}

De forma geral, observou-se que os medidores eletrônicos de pressão e vazão, mesmo que de baixo custo, apresentaram medições confiáveis, encorajando suas aplicações não somente em ensaios de bancada, como também em aplicações em sistemas reais.

Além disso, a variação de pressão causada pela alteração do número de bicos em operação na barra de pulverização é evidente e a inserção da válvula como dispositivo regulador de vazão e, consequentemente, de pressão mostrou-se uma alternativa viável.

Nesse contexto, para trabalhos futuros, sugere-se a adição da válvula de controle na modelagem matemática do sistema (Equação 13) e, assim, realização de estudos e aplicações de técnicas de controle em malha fechada que atendam às condições de trabalho requeridas.

Ainda como trabalhos futuros, sugere-se a obtenção de curvas para outros tipos de bicos de pulverização e também para barras com um número maior de bicos, de modo a avaliar outros pontos de operação dos sistemas reais.

Dessa forma, este trabalho contribui para a comunidade acadêmica no que tange à inserção de conteúdos na área discutida, até então ausentes ou pouco abordados em trabalhos científicos.

Além disso, na prática, o sistema estudado pode auxiliar na implementação em pulverizadores agrícolas de pequenas propriedades rurais, contribuindo para a estabilização da gota e, consequentemente, para que haja efetiva redução no uso dos agrotóxicos por parte dos agricultores e no consumo destes por parte da população.

\section{AGRADECIMENTOS}

Agradece-se à Fundação de Apoio e Amparo à Pesquisa do Estado do Rio Grande do Sul (FAPERGS) projeto número
17/2551- 0000896-0 pelo suporte financeiro. Agradecese, também, ao Prof. Dr. André Oldoni (IFSUL) e aos integrantes do projeto AutoCERES pelo apoio, incentivo e auxílio nas etapas de desenvolvimento desta pesquisa.

\section{REFERÊNCIAS}

Araújo, N. (2016). Agrishow 2016 - jacto lança dois pulverizadores automotrizes. Successful Farming.

Bega, E.A., Délmee, G.J., Cohn, P.E., Bulgarelli, R., Koch, R., and Finkel, V.S. (2006). Instrumentação Industrial. Editora Interciência, Rio de Janeiro, 2 edition.

Deere, J. (2018). Tecnologia em agricultura de precisão. John Deere.

Delmée, G.J. (2003). Manual de Medição de Vazão. Editora Blucher, São Paulo, 3 edition.

Felizardo, K.R., Mercaldi, H.V., Cruvinel, P.E., Oliveira, V.A., and Steward, B.L. (2016). Modeling and model validation of a chemical injection system. American Society of Agricultural and Biological Engineers.

Garcia, C. (2005). Modelagem e Simulação de Processos Industriais e de Sistemas Eletromecânicos. Editora da Universidade de São Paulo, São Paulo, 2 edition.

Grützmacher, D.D., Grützmacher, A.D., Agostinetto, D., Loeck, A.E., Roman, R., Peixoto, S.C., and Zanella, R. (2008). Monitoramento de agrotóxicos em dois mananciais hídricos no sul do brasil. Revista Brasileira de Engenharia Agrícola e Ambiental, 12(6), 632-637.

Holterman, H.J., Zande, J.C.v.d., Porskamp, H.A.J., and Huijsmans, J.F.M. (2016). Modelling spray drift from boom sprayers. American Society of Agricultural and Biological Engineers.

Hughes, T.A. (2002). Measurement and Control Basics. ISA Press, Research Triangle Park, 3 edition.

IBGE (2017). Censo Agropecuário 2017. Instituto Brasileiro de Geografia e Estatística.

ISA (2009). ISA 5.1: Instrumentation Symbols and Identification. International Society of Automation.

Mercaldi, H.V., Peñaloza, E.A.G., Mariano, R.A., and Cruvinel, P.E. (2017). Flow and pressure regulation for agricultural sprayers using solenoid valves. International Federation of Automatic Control.

Milhorance, F. (2015). Brasil lidera o ranking de consumo de agrotóxicos. O Globo.

Newlands (2019). Safe Application Of Pesticides Boom Sprayers. Disponível em: https://www. newlandstraining.co.uk/pesticide-application/ fepa-spraying/fepa-spraying-boom-spraying/. Acesso em: 22/04/2019.

Otmis, J. (2017). Controle bico a bico omni 700. Jacto Otmis.

Ribeiro, M.L., Lourencetti, C., Pereira, S.Y., and de Marchi, M.R.R. (2007). Contaminação de águas subterrâneas por pesticidas: avaliação preliminar. Química Nova, 30(3), 688-694.

Steward, B.L. and Humburg, D.S. (2000). Modeling the raven scs-700 chemical injection system with carrier control with sprayer simulation. American Society of Agricultural Engineers, 43(2), 231-245.

Tip, F. (2018). Bico de pulverização hypro fantip. Fan Tip. 